\title{
Immunodiagnosis Tools for Parasitic Diseases
}

Deepak $\mathbf{S}^{1 *}$ and Singla LD $^{2}$

${ }^{1}$ Department of Veterinary Parasitology, Institute of Veterinary Education and Research, India

${ }^{2}$ Department of Veterinary Parasitology, Guru Angad Dev Veterinary and Animal Sciences University, India

\begin{abstract}
Regarding harmful agents in livestock, parasites play an important role moreover it also effect $1 / 4^{\text {th }}$ of the human populace by various zoonotic diseases (e.g. Toxplasma, Cryptosporidium, Trypanosomiasis, etc.). In livestock the parasites successful invade host immune responses, so early diagnosis of parasitic agents by various immunodiagnostic techniques is very important. Numerous immunological/serological techniques have been emerged such as the complement fixation test (CFT), immunodiffusion (ID), indirect haemagglutination (IHA), indirect immunofluorescent antibody test (IFA), various forms of enzyme-linked immunosorbent assay (ELISA) [Sandwich ELISA, indirect and direct ELISA, competitive ELISA, falcon assay screening test ELISA (FAST-ELISA), dot-ELISA, rapid antigen detection system (RDTS), and luciferase immune precipitation system (LIPS)] and radioimmunoassay (RIA). They targets different components of parasite, moreover they can detect disease before the emergence of clinical sign. These tests are used for many important parasitic diseases like Echinococcus multilocularis, Wuchereria bancrofti, Taenia solium and parasite causing Babesiosis, Toxoplasmosis, Visceral leishmaniasis, Human African Trypanosomiasis in both man and animals. Moreover now a day's nano and biosensor technology has also been used for improvising the diagnostic aspect. This current mini-review is an attempt to consolidate some information of different serological based test for early diagnosis of main parasitic disease.
\end{abstract}

Keywords: Parasite; Immunodiagnostic techniques; Immune system; Zoonotic disease

\section{Introduction}

According to World Health Organization (WHO) population of world will reach about 9.7 billion by 2050 . So along with agricultural food, animal food is also required as alternate food resource. Health along with production of animals is affected by various parasitic diseases. It was estimated that only with a $6 \%$ reduction in animal disease could provide food for an additional 250 million people [1]. Parasite are divided mainly into different groups i.e. trematodes (flukes), cestode (flat worm), nematodes (round worm), arthropods and protozoan. Most of the parasites have a 2 host, prey predator life cycle, one act as intermediate host while other is a definitive host. All cause some of the most devastating and prevalent diseases in both humans and animals. So there timely detection is very important aspect to reduce the loss of livestock's health and production [1]. Simple microscopic detection has many limitations so immunological detection plays a vital role as it is based on antibodies detection. Numbers of immunological/ serological techniques have been emerged such as the complement fixation test (CFT), immunodiffusion (ID), indirect haemagglutination (IHA), indirect immunofluorescent antibody test (IFA),various forms of enzyme-linked immunosorbent assay (ELISA) [Sandwich ELISA, indirect and direct ELISA, competitive ELISA, falcon assay screening test ELISA (FAST-ELISA), dot-ELISA, rapid antigen detection system (RDTS), and luciferase immunoprecipitation system (LIPS)] and radioimmunoassay (RIA). In RIA basic immunoassay principles were established some 30 years ago in studies on binding of radiolabelled insulin to antibodies to insulin (anti-insulin antibodies) [2]. Less frequently used tests include card agglutination, latex agglutination and capillary agglutination. Almost all of these tests are based on the reaction of antibodies with antigenic parasite components (whole or soluble) resulting in antigen-antibody complexes. The complexes thus formed are detected by the addition of antiglobulins coupled to fluorescein and rhodamine dyes, radioisotopes or enzymes.

\section{Some tests which are routinely used for parasite detection}

- Complement fixation test (CFT): Trypanosomiasis, Helminthiasis, Anaplasmosis, Babesiosis, Toxoplasmosis.
- Rapid card agglutination test (CAT): Anaplasmosis, Babesiosis, Trypanosomiasis.

- Indirect fluorescent antibody test (IFA): Theileriosis, Helminthiasis, Anaplasmosis, Besnoitiosis, Ehrlichiosis/ Cowdriosis, Malaria, Babesiosis, Trypanosomiasis, Toxoplasmosis.

- Radioimmunoassay (RIA): Babesiosis.

- ELISA: Babesiosis, Besnoitiosis, Helminthiasis, Toxoplasmosis, Trypanosomiasis Anaplasmosis, Ehrlichiosis/Cowdriosis [3].

\section{Different parasite targeted by immunodiagnosis techniques}

Echinococcus multilocularis: Cystic echinococcosis (CE) and Alveolar echinococcosis (AE) is a severe zoonotic disease caused by the larval stage (metacestode) of the helminth Echinococcus multilocularis. By affinity chromatography a native Em2 antigen (also termed Em2a) was purified originally from E. multilocularis metacestode tissue extracts [4]. By immunoprecipitation, western blot and ELISA it was demonstrated that it was an E. multilocularis-specific antigen with apparent mass of $54 \mathrm{kDa}$. Both in vivo and in vitro studies revealed that $\mathrm{Em} 2$ is a structural component found only in the metacestode laminated layer, and not in freshly hatched oncospheres, protoscolices or adult stages [5]. Moreover a glycosylated antigen Em2 (G11) has been found to be the major antigenic component of Em2 [5]. Walker et al. identified an Em492 in E. multilocularis metacestode [6]. Both

*Corresponding author: Deepak S, Department of Veterinary Parasitology, Institute of Veterinary Education and Research, India, Tel: +91-9906070221; E-mail: deepak_sumbria@yahoo.com

Received November 10, 2016; Accepted December 26, 2016; Published December 30, 2016

Citation: Deepak S, Singla LD (2016) Immunodiagnosis Tools for Parasitic Diseases. J Microb Biochem Technol 8: 514-518. doi: 10.4172/1948-5948.1000336

Copyright: @ 2016 Deepak S, et al. This is an open-access article distributed under the terms of the Creative Commons Attribution License, which permits unrestricted use, distribution, and reproduction in any medium, provided the original author and source are credited. 
the antigens are related immunologically because both share the galactose-a $(1,5)$-galactose epitope. In protoscolices of Echinococcus granulosus homologous component of Em492, termed E4+, had also been identified. E4+ has being involved in the modulation of the cellular immune response in experimental mice infections by stimulating interleukin (IL)-10 secretion and subsequent induction of the type 2 cytokine response. Other E. granulosus antigens with wellcharacterised glycosylate moieties include the carcinoma associated $\mathrm{Tn}$ antigen, hydatid cyst fluid antigen 5 and the blood group P1 epitope $[6,7]$. However, to our knowledge, none of these components has been described in E. multilocularis.

In alveolar echinococcosis infection the test based on sero-diagnosis mainly are based on native and recombinant antigens of the Echinococcus multilocularis metacestode such as Em2, Em2plus, Em2plus ELISA, CH-10, N3C, pAP, Em70, Em90, rII/3, rII/3-10 and rEM10. From protoscolex a crude E. multilocularis antigen was extracted and a native EM2 protein was identified as a $70 \mathrm{kDa}$. Two new polypeptides of the E. multilocularis metacestode, referred as to Em70 and Em90, have also been identified as potential antigens for the serodiagnosis of AE. For the sero-diagnosis of AE now a day's test mainly based on native and recombinant antigens of the Echinococcus multilocularis protoscolex and/or adults such as rEM4, EM13, Em18,16, Em18, rEm18, rEm18$1, \mathrm{rEm} 18-2$ are used [8]. For effective discrimination between $\mathrm{AE}$ and $\mathrm{CE}$ patients the first echinococcus component used was Em2. Now a day's at least one western blot kit (Echinococcus Western Blot IgG; LDBIO Diagnostics, Lyon, France) is available commercially for routine serological diagnosis and differentiation of Echinococcus spp. This test, based on the detection of specific IgG directed against E. multilocularis whole larval antigen (both Em16 and Em18 antigens) allows correct differentiation between $\mathrm{AE}$ and CE patients in $76 \%$ of cases, thereby achieving similar diagnostic sensitivities to those reported previously for the Em2plus ELISA assay.

Main antigen targeted for serological tests differentiation between alveolar and cystic echinococcosis are Em1+Em2, Em18+Ag B, 16, 18, 27b kD, 18 kD, Em18, EmII/3, rEM10+rEG55, rEm18 [8]. Main copro-antigen targeted for sero-testing characteristics for Echinococcus multilocularis infection in definitive hosts are Anti-E.g (E. granulosus). ESP Ad (excretory secretory product of adults), Anti-E.m (Echinococcus multilocularis). S. Ad. Anti-E.m. S (sensitivity). Ad. mAb (monoclonal antibodies) Em9, Anti-E.m. S. Ad, Chekit Echino test [8].

Wuchereria bancrofti: Bancroftian filariasis is an infectious disease produced in man by the filarial parasite, i.e., Wuchereria bancrofti. In bancroftian filariasis serum immunoglobulins IgE and IgG were found to be at elevated level compared to controls. By solid phase radioimmunology assay the specific IgE antibody levels were found to be lowered in chronic filariasis and ELISA using Brugia malayi and W. bancrofti antigens respectively. Homologous and Heterologous antigens are also been used for its effective immunodiagnosis. Research targeting the utility of $W$. bancrofti microfilarial antigens in gel diffusion (GD), indirect haemagglutination test (IHAT), counter immuno electrophoresis (CIE), indirect fluorescent antibody test (IFAT) and ELISA for detection of filarial antibody in filarial sera is going on. For the detection of antibody GD was found to be least sensitive but on the other hand ELISA was observed to be highly sensitive. Using soluble W. bancrofti micro filarial antigen, the efficiency of three tests (IHAT, IFAT and ELISA) was compared for the detection of antibody. Filarial antibody could be detected in $93 \%, 100 \%, 81 \%$ of the microfilaraemics, $75 \%, 90 \%, 100 \%$ of chronic pathology and none of the non-endemic sera by IHAT, IFAT and ELISA respectively.
Excretory-secretory (ES) antigens: By maintaining W. bancrofti microfilariae (mf) in medium 199 (3-4 thousand $\mathrm{mf} / \mathrm{ml}$ of medium) supplemented with organic acids and sugars of Grace's medium but without serum, the $W$. bancrofti microfilarial (mf) excretory-secretory (ES) antigens were obtained. Utility of W. bancrofti ES antigen has also been explored in IHAT, Penicillinase ELISA and CNBr-Sepharose IFAT for detection of filarial antibody. By fractionation of ES antigens with the help of membrane filtration an ES4 antigen fraction (which was found to be a glycoprotein in nature and was highly reactive) microfilaraemia sera was obtained. W. bancrofti $\mathrm{mf}$ ES antigen specific IgE antibody was detected in filariasis and tropical eosinophilia by immunofluorescence assay and ELISA [8]. These studies showed the detection of ES antigen specific $\operatorname{IgG}+\mathrm{A}+\mathrm{M}$ antibodies. Circulating filarial antigen was concentrated from microfilaraemia plasma by salt precipitation and was identified as an antigen of microfilarial origin using anti rabbit $\mathrm{mf}$ sera in CIE [9]. Under field condition for the detection of monoclonal antibody of the circulating antigen of $W$. bancrofti adult worm ICT Filariasis, a rapid card test format was also evaluated [10]. Results concluded that it has high sensitivity, lack of cross-reactions, single reagent and rapidity of the test so, ICT can be recommended for screening of Bancroftian filariasis. In Egypt for lymphatic filariasis AMRAD-ICT-Fil was evaluated in 1813 endemic and 102 non endemic participants [10]. The infection rates detected were $9 \%$ by AMRADICT-Fil, $8.8 \%$ by ELISA, $3.5 \%$ by membrane filtration and $2.8 \%$ by thick smear. In Sri Lanka the sensitivity, specificity and cost effectiveness of the same test card was estimated against thick blood film (TBF) and nuclepore membrane filtration (NMF). Results depicted that it is the most effective (both sensitive and specific) its cost is very high [10].

Babesiosis: To differentiate B. equi-infected horses from B. caballiinfected and uninfected horses Babesia Immuno Chromatic test (ICT) for the rapid detection of antibodies for B. equi was developed. Using a recombinant $B$. caballi $48 \mathrm{kDa}$ rhoptry protein $(\mathrm{rBc} 48)$ and a recombinant truncated $B$. equi merozoite antigen 2 (rEMA-2t) for the simultaneous detection of Babesia caballi- and B. equi-specific antibodies (BceICT) was developed by same investigators in 2006 [10]. In dogs for the detection of antibodies to B. gibsoni, ICT was developed and evaluated with sera using recombinant truncated P50 (P50t). Kim and his co-workers developed two ICTs with nitrocellulose membrane based immunoassays for rapid serodiagnosis of bovine babesiosis caused by B. bovis (BoICT) and B. bigemina (BiICT) [10].

Toxoplasmosis: In cats and mice for detection of antibodies against Toxoplasma gondii ICT was applied using recombinant truncated surface antigen- 2. Under field condition this test is simple, rapid, accurate and relatively inexpensive $[9,10]$.

Visceral leishmaniasis: For visceral leishmaniasis (VL) under field conditions an ICT targeting leishmanial antigen K39 by antigenimpregnated nitrocellulose paper strips was adapted [10]. On strip a drop of serum and/or peripheral blood is applied followed by buffer resulted the formation of two visible bands indicating the presence of anti-k39 IgG. Moreover it was reported that ICT K26 was equally specific but less sensitive than ICT K39. These two antigens increased the overall sensitivity of the test by complementing each other [10]. In India, Bangladesh and Brazil the usefulness of ICT rK39 was assessed in diagnosis of VL. Moreover in post Kala-azar dermal leishmaniasis (PKDL) sera ICTrk39 was also evaluated. For PKDL the strip test depicted $91 \%$ sensitivity and 100\% specificity [10]. In India anti-K39 ICT (IT-Leish; DiaMed AG) was proved to be highly sensitive and specific for the serod-testing of VL. Later on it was concluded that ICT strip test is easy, quick, requires no technical facilities with higher 
sensitivity and specificity entailing it to be the ideal test for the diagnosis of kala-azar in field level [10].

Regarding some other important haemo-protozoan disease ELISA has been used as superior test in South American trypanosomiases filariasis and schistosomiasis [11-13]. ELISA is not yet as specific or sensitive as immunofluorescent methods in malarial antibody tests probably because the antigen preparations are still crude. In some other parasitic disease it was seen that purification of antigens remains an obstacle for serodiagnos is in all these conditions but is rapidly progressing; for instance, the purification of schistosoma-egg antigen should further increase the test's specificity and sensitivity in this disease $[11,14]$. Evaluation of the ELISA enzyme-linked procedure is not yet complete in some other protozoal infections, but current methods such as counter electrophoresis in occult amoebic abscess or immunofluorescent methods in giardiasis yield acceptable results $[15,16]$.

Human African trypanosomiasis: In region like sub-Saharan Africa a vector-borne parasitic disease Human African Trypanosomiasis (HAT), or sleeping sickness, is endemic [17]. As we all know this disease is caused by an extracellular parasite called Trypanosoma (genus). Many serological tests has been used for its diagnosis like-

a. CATT (Card-Agglutination Trypanosomiasis Test)- CATT is an antigen-antibody based test, useful for initial population screening to identify suspected cases. The test was developed in the late 1970s. It can be carried out on blood, capillary blood obtained from a finger prick, or blood from impregnated filter papers [18]. Antigen used for the test CATT is complete bloodstream forms of Trypanosoma bruce gambiense variable antigen type LiTat 1.3 and for T. evansi it is RoTat 1.2. The sensitivity of CATT on blood is about $91 \%$, with a range of $78-99.8 \%$, and negative predictive values as high as $99-100 \%$ has been reported in mass population screening $[19,20]$.

b. Cytokines and Chemokines- The level of cytokines and chemokines has been measured for the investigation of their diagnostic potential both in T. b. gambiense and T. b. rhodesiense. The most interesting cytokines and chemokines used for staging sleeping sickness are IL-10, IL-6, IL-1 $\beta$, CCL-3, CXCL-8, SLPI, Lipocalin 2, ICAM-1, VCAM, MMP-9, MMP-2, CXCL-10 and CXCL-13, A recent study initially evaluated the most promising molecules such as CXL-10, CXCL-13, ICAM- 1, VCAM-1, IgM, MMP-9 and B2MG and confirmed their capacity to act as accurate staging markers [21-23].

c. Proteomics- Another approach currently under investigation is the evaluation of the changes in protein expression between pathological and healthy conditions. Only a few studies have established first and second stage HAT disease CSF protein profiles. Two of these proteins, osteopontin and beta-2microglobulin, were confirmed to be accurate markers of first and second stage patients [24]. It is important to research and study new protein biomarkers, particularly for discriminating stage 2 and stage 1 of the disease, and this is possible thanks to progress in matters of protein and peptide analysis with the evolution of mass spectrometry [25].

d. Polysomnography- In recent years, research has been conducted on the most typical clinical manifestation of HAT: the alteration of the normal sleep-wake cycle [26]. Studies show a high number of Sleep Onset Rapid Eye Movement Periods (SOREMP) in stage 2 patients during their sleep, not only restricted to nighttime, but also during daytime sleep too. Treatment with Melarsoprol seems to reduce the appearance of SOREMPs.

Regarding chagas disease the immunoblot assays have been also conducted; among the various immunological techniques the most important is TESAblot that consists of detection of antibodies against the antigens TESA (trypomastigote excreted-secreted antigen). This assay is currently commercialized and has been widely used because of its high sensitivity and specificity and ability to resolve doubtful serology and cross-antigenicity issues with related protozoan parasites in regions where the disease is endemic [27-31]. The TESA antigen assay also has been used for other tests like ELISA [32].

Neurocysticercosis (NCC): Immunodiagnosis of NCC can be done by two ways: by identifying antibodies against cysticercal antigens or by identifying parasites antigens directly. The source of antigens for immunodiagnosis has been commonly from Taenia solium (the parasite responsible for NCC) but related species as Taenia crassiceps, Taenia saginata or Taenia taeniformis have also been used as antigen sources. Now a days multiple antigens have been used and among them, main are low molecular mass (LMM) antigens, excretory/secretory (ES), crude soluble extract (CSE), total saline extract, antigen B, lentil lectin glycoproteins (LLGPs), vesicular fluid (VF), membrane and scolex extracts, somatic antigens, recombinant proteins, and synthetic peptides. Multiple methods used to date for the immunodiagnosis of NCC like complement fixation, agglutination, radio immune assays, ELISA and Western Blot (WB) can be counted [33]. LLGP-WB has a sensitivity of $>90 \%$ and a specificity of $100 \%$. In Indian patients almost two thirds of the NCC patients have an SCG; LLGPs have shown to be less sensitive than for multiple cysticerci. Antigen for immunodiagnosis from the members of the $8 \mathrm{kDa}$ family, Ts $18 \mathrm{var} 1$ has been produced in insect cells as well as TsRs1, Ts18var1, and Ts18 Var3; the 14 and $18 \mathrm{kDa}$ proteins produced by recombination; Ts14, Ts18var1, TSRS1 and TSRS2var1 by chemical synthesis, and full-length Ts18 and Ts14 by chemical ligation, Ag1V1/Ag2 by recombination as well as Ts8B1, Ts8B2, Ts8B3, Ts 14 and a $10 \mathrm{kDa}$ protein, GP50, which is not a member of the $8 \mathrm{kDa}$ family but it is part of the LLGPs, was produced by recombination in bacteria and in a baculovirus expression system [3339]. Outside from LLGPs other proteins that have also been produced or synthesized include T24 (integral membrane protein that does not bind to lentil lectin) produced in a drosophila cell line; HP6-Tsag (oncospheral adhesion protein of Taenia saginata) in bacteria and baculovirus systems with similar specificities between the systems (9395\%), but higher sensitivity for the inactive cases by the baculovirus protein (48-64\%); peptide NC-1 selected by phage-display; peptides KETc12, 410 and 413 synthesized from a cDNA library of T. crassiceps and recombinant TS24 and Es33. The methods of production are varied, as well as the results and the ways to evaluate the produced protein, some giving very good sensitivities but in other cases, the native protein is much better than the produced one [33].

Use of nanotechnology for the effective diagnosis of parasitic disease: Nanotechnology exactly means any technology performed on a nano-scale that has practical applications to our every-day occurring daily activities. This can offer an improvement in imaging and diagnosis of the fatal parasitic diseases as well, slipping away the limitations of some biological barriers. In the parasitology field the use nanotech have only a few applications and the research have been reported on Leishmania sp. and Plasmodium sp. [40,41]. Nano-particles have also shown improvement in the bioavailability and drug selectivity, even in very complicated treatments like cerebral malaria by Plasmodium falciparum infection [41]. Some nano-partical like Pegylated or polyplex offer fantastic options to increase the biodistribution of a 
compound. In veterinary medicine it may also help in mass treatments of ectoparasites, facilitating the drug-parasite interaction. Moreover the effectiveness of phytotherapeutic products can be improved, like the recent treatment of antiparasitic silver nano-particles using Cissus quadrangularis against Hippobosca aculata and Rhipicephalus (Boophilus) microplus [42]. Nano-devices also help veterinarians to suggest a correct health management [43]. In Cuba and Brazil some project are currently running using nanopeptides as a preliminary test against ruminant helminths (Haemonchus contortus and Fasciola hepatica). This technology can also allow the development of adjuvants for veterinary parasite vaccines, boosting their present low efficacy. Newly, a nano-microparticulated malaria vaccine elicited long-lasting protective antibody titers with only a single dose [44].

Biosensor: A biosensor is an analytical device that converts molecular recognition of a target analyte into a measurable signal via a transducer. Common to all such instruments is a support material, on which one of two affinity-pairing partners (the recognition element) is immobilized. The partners may be an enzyme and its substrate, an antibody/antigen pair, a receptor and its specific ligand, or even living cells and an analyte that binds specifically to them. Bioreceptor types used in biosensors are as follows: Nucleic acid, Enzyme, Whole-cell, Antibody and receptor, Micro and nano and biomimetic $[45,46]$.

For parasitic diseases, biosensors offer the possibility of an easyto-use, sensitive and inexpensive technology platform that can identify parasite rapidly and predict effective treatment $[47,48]$. It has many advantages which include small fluid volume manipulation (less reagent and lower cost), short assay time, low energy consumption, high portability, high through put and multiplexing ability $[49,50]$. For the detection of malaria various biomarkers have been used among them the plasmodial lactate dehydrogenase and histidinerich protein II (HRP II) has received increasing attention [51]. The widely used rapid detection tests (RDTs) for malaria succumb to many drawbacks which promotes exploration of more efficient economical detection techniques. A low-cost biosensor system was also made with nanostructured films containing specific Leishmania amazonensis and T. cruzi antigens and employing impedance spectroscopy as the detection method [52,53].

\section{Conclusion}

Concise information provided in this mini-review regarding the progress in the field of diagnostic test in various parasitic diseases of livestock and humans may be a guideline for veterinarian clinician and academician.

\section{References}

1. Ristic M, Montenegro-James S (1987) Progress in the immunoprophylaxis of hemoparasitic diseases of cattle. Agribus Worldwide 19: 9-10.

2. Berson SA, Yallow RS, Bauman A, Rothschild MA, Newerly K (1956) Insulin-i metabolism in human subjects: demonstration of insulin binding globulin in the circulation of insulin treated subjects. J Clin Invest 35: 170-190.

3. Ambrosio RE, Waal DTD (1990) Diagnosis of parasitic disease. Rev sci tech Off int Epiz 9: 759-778.

4. Gottstein B (1985) Purification and characterization of a specific antigen from Echinococcus multilocularis. Parasite Immunol 7: 201-212.

5. Deplazes P, Gottstein B (1991) A monoclonal antibody against Echinococcus multilocularis Em2 antigen. Parasitology 103: 41-49.

6. Walker M, Baz A, Dematteis S, Stettler M, Gottstein B, et al. (2004) Isolation and characterization of a secretory component of Echinococcus multilocularis metacestodes potentially involved in modulating the host-parasite interface. Infect Immun 72: 527-536.
7. Makni S, Ayed KH, Dalix AM, Oriol R (1992) Immunological localization of blood $\mathrm{pl}$ antigen in tissues of Echinococcus granulosus. Ann Trop Med Parasitol 86: 87-88.

8. Carmena D, Benito A, Eraso E (2007) The immunodiagnosis of Echinococcus multilocularis infection. Clin Microbiol Infect 13: 460-475.

9. Sumbria D, Singla LD (2015) Mammalian parasitic vaccine: A consolidated exposition. J Vaccines Immun 1: 50-59.

10. Abaza SM (2008) Immunochromatographic assays in diagnosis of parasitic diseases. Parasitol United J 1: 1-13.

11. Spencer HC, Allain DS, Sulzer AJ, Collins WE (1980) Evaluation of the microenzyme-linked immunosorbent assay for antibodies to Trypanosoma cruzi. Am Trop Med Hyg 29:179-182.

12. Ambroise-Thomas P, Filariasis (1980) In: Houba V, ed. Immunological investigation of tropical parasitic disease. Edinburgh: Churchill Livingstone 94.

13. Deelder AM, Ruittenberg EJ, Kornelis D, Steerenberg PA (1977) Schistosoma mansoni; comparison of the immunoperoxidase techniques DASS and ELISA for human diagnosis. Exp Parasitol 41:133-140.

14. McLaren ML, Lillywhite JE, Dunne DW, Doenhoff MJ (1981) Serodiagnosis of human Schistosoma mansoni infections: enhanced sensitivity and specificity in ELISA using a fraction containing S. mansoni egg antigens $\mathrm{xl}$ and ao. Trans $\mathrm{R}$ Soc Trop Med Hyg 75: 72-79.

15. Tosswill JHC, Ridley DS, Warhurst DC (1980) Counter immunoelectrophoresis as a rapid screening test for amoebic liver abscess. Clin Pathol 33: 33-35.

16. Visvesvara GS, Smith PD, Healy GR, Brown WR (1980)An immunofluorescence test to detect serum antibodies to Giardia lamblia. Ann Initern Med 93: 802-805.

17. Bonnet J, Boudot C, Courtioux B (2015) Overview of the diagnostic methods used in the field for human african trypanosomiasis: What could change in the next years? BioMed Res Int 583262: 10.

18. Penchenier L, Gr'ebaut P, Njokou F, Eyenga Eboo V, Uscher PB (2003) Evaluation of LATEX/T. b. gambiense for mass screening of Trypanosoma brucei gambiense sleeping sickness in central Africa. Acta Trop 85: 31-37.

19. Ebeja AK (2013) Journ'ee scientifique THA `a Kinshasa. Bulletin HAT platform, capacities/newsletter.final.

20. Checchi F, Chappuis F, Karunakara U, Priotto G, Chandramohan D (2011) Accuracy of five algorithms to diagnose gambiense human African trypanosomiasis. PLoS Neg Trop Dis 5: 1233.

21. Tiberti N, Hainard A, Sanchez JC (2013) Translation of human African trypanosomiasis biomarkers towards field application. Trans Prot 1: 12-24

22. Courtioux B, Pervieux L, Vatunga G, Marin B, Josenando T et al, (2009) Increased CXCL-13 levels in human African trypanosomiasis meningoencephalitis. Trop Med Int Health 14: 529534

23. Kristensson K, Nygard M, Bertini G, Bentivoglio M (2010) African trypanosome infections of the nervous system: Parasite entry and effects on sleep and synaptic functions. Pro Neurobio 91: 152-171.

24. Tiberti N, Hainard A, Lejon V, Robin X, Ngoyi DM, et al. (2010) Discovery and verification of osteopontin and beta-2-microglobulin as promising markers for staging human African trypanosomiasis. Mol Cell Proteomics 9: 2783-2795.

25. Geiger A, Simo G, Grebaut P, Peltier JB, Cuny G, et al. (2011) Transcriptomics and proteomics in human African trypanosomiasis: Current status and perspectives. J Proteomics 74: 1625-1643.

26. Brun R, Blum J, Chappuis F, Burri C (2010) Human African trypanosomiasis The Lancet 375: 148-159.

27. Ramírez JD, Guhl F, Umezawa ES, Morillo CA, Rosas F, et al. (2009) Evaluation of adult chronic Chagas' heart disease diagnosis by molecular and serological methods. J Clin Microbiol 47: 3945-3951.

28. Umezawa ES, Nascimento MS, Kesper N Jr, Coura JR, Borges-Pereira J, et al (1996) Immunoblot assay using excreted-secreted antigens of Trypanosoma cruzi in serodiagnosis of congenital, acute and chronic Chagas' disease. J Clin Microbiol 34: 2143-2147.

29. Umezawa ES, Bastos SF, Camargo ME, Yamauchi LM, Santos MR, et al (1999) Evaluation of recombinant antigens for serodiagnosis of Chagas disease in South and Central America. J Clin Microbiol 37: 1554-1560.

30. Umezawa ES, Souza Al, Pinedo-Cancino V, Marcondes M, Marcili A, et al. (2009) TESA-blot for the diagnosis of Chagas disease in dogs from co-endemic 
Citation: Deepak S, Singla LD (2016) Immunodiagnosis Tools for Parasitic Diseases. J Microb Biochem Technol 8: 514-518. doi: 10.4172/19485948.1000336

regions for Trypanosoma cruzi, Trypanosoma evansi and Leishmania chagasi. Acta Trop 111: 15-20.

31. Hernández, Ramírez (2013) Molecular diagnosis of vector-borne parasitic diseases. Air water borne diseases 2: 1 .

32. Lockwood DN, Sundar S (2006) Serological tests for visceral leishmaniasis. BMJ 333: 711-712.

33. Esquivel-Vel 'azquez M, Ostoa-Saloma P, Morales-Montor J, Hern'andez-Bello $\mathrm{R}$, Larralde C (2011) Immunodiagnosis of neurocysticercosis: Ways to focus on the challenge. J Biomed Biotechnol.

34. Bueno EC, Scheel CM, Vaz AJ, Machado LR, Livramento JA, et al. (2005) Application of synthetic $8 \mathrm{kD}$ and recombinant GP50 antigens in the diagnosis of neurocysticercosis by enzyme-linked immunosorbent assay. Am J Trop Med Hyg 72: 278-283.

35. da Silva MRM, Maia AAM, Esp' indola NM, Machado LDR, Vaz AJ, et al. (2006) Recombinant expression of Taenia solium TS14 antigen and its utilization for immunodiagnosis of neurocysticercosis. Acta Trop 100: 192-198.

36. Scheel CM, Khan A, Hancock K, Garcia HH, Gonzalez AE, et al. (2005) Serodiagnosis of neurocysticercosis using synthetic $8 \mathrm{KD}$ proteins: Comparison of assay formats. Am J Trop Med Hyg 73: 771-776.

37. Lee EG, Lee MY, Chung JY, Je EY, Bae YA, et al. (2005) Feasibility of baculovirus-expressed recombinant 10-kDa antigen in the serodiagnosis of Taenia solium neurocysticercosis. Trans R Soc Trop Med Hyg 99: 919-926.

38. Chung JY, Bahk YY, Huh S, Kong SY, Kong Y, et al. (1999) A recombinant 10-kDa protein of Taenia solium metacestodes specific to active neurocysticercosis. J Infect Dis 180:1307-1315.

39. Harinath BC (1984) Immunodiagnosis of Bancroftian filariasis-Problems and progress. J Biosci 6: 691-699.

40. Carvalho RF, Ribeiro IF, Miranda-Vilela AL, Souza Filho J, Martins OP, et al. (2013) Leishmanicidal activity of amphotericin B encapsulated in PLGA-DMSA nanoparticles to treat cutaneous leishmaniasis in C57BL/6 mice. Exp Parasitol 135: $217-222$

41. Waknine-Grinberg JH, Even-Chen S, Avichzer J, Turjeman K, BenturaMarciano A, et al. (2013) Glucocorticosteroids in nano-sterically stabilized liposomes are efficacious for elimination of the acute symptoms of experimental cerebral malaria. PLoS ONE 8: 2722.

42. Santhoshkumar T, Rahuman AA, Bagavan A, Marimuthu S, Jayaseelan C, et al (2012) Evaluation of stem aqueous extract and synthesized silver nanoparticles using Cissus quadrangularis against Hippobosca maculata and Rhipicephalus (Boophilus) microplus. Exp Parasitol 132: 156-165.

43. Cruz AA, Molento MB (2015) Nanotechnology: Meeting the future of Veterinary Parasitology Research. Pesquisa Veterinária Brasileira 35: 842-843.

44. Dinglasan RR, Armistead JS, Nyland JF, Jiang X, Mao HQ (2013) Single-dose microparticle delivery of a malaria transmission-blocking vaccine elicits a longlasting functional antibody response. Curr Mol Med 13: 479-487.

45. Wang J (2015) Nanomaterial-based electrochemical biosensors. Analyst 131 421-426.

46. Shipway AN, Lahav M, Willner I (2011) Nanostructured gold colloid electrodes. Adv Mater 12: 993-998.

47. Foudeh AM, Fatanat Didar T, Veres T, Tabrizian M (2012) Microfluidic designs and techniques using lab-on-a-chip devices for pathogen detection for point-ofcare diagnostics. Lab Chip 12: 3249-3266.

48. D'orazio P (2011) Biosensors in clinical chemistry-2011 update. Clin Chim Acta 412: $1749-1761$

49. Whitesides GM (2006) The origins and the future of microfluidics. Nature 442 368-373.

50. Sin MY, Mach KE, Wong PK, Liao JC (2014) Advances and challenges in biosensor-based diagnosis of infectious diseases. Expert Rev Mol Diagn 14: 225244.

51. Jain P, Chakma B, Patra S, Goswami P (2014) Potential biomarkers and their applications for rapid and reliable detection of malaria. BioMed Res Int 852645: 20.

52. Hommel M, Peters W, Ranque J, Quilici M, Lanotte G (1978) The microELISA technique in the serodiagnosis of visceral leishmaniasis. Ann Trop Med Parasitol 72: 213-218.

53. Perinoto AC, Maki RM, Colhone MC, Santos FR, Migliaccio V, et al. (2010) Biosensors for efficient diagnosis of leishmaniasis: Innovations in bioanalytics for a neglected disease. Anal Chem 82: 9763-9768. 